\title{
GYY4137 Attenuates Sodium Deoxycholate-Induced Intestinal Barrier Injury Both In Vitro and In Vivo
}

\author{
Zeyang Chen, Jianqiang Tang, Pengyuan Wang $(\mathbb{D}$, Jing Zhu, and Yucun Liu \\ Division of General Surgery, Peking University First Hospital, Peking University, 8 Xi Shiu Street, Beijing 100034, China \\ Correspondence should be addressed to Yucun Liu; yucun_liu@126.com
}

Received 4 July 2019; Accepted 10 September 2019; Published 13 October 2019

Academic Editor: Christina Pabelick

Copyright (c) 2019 Zeyang Chen et al. This is an open access article distributed under the Creative Commons Attribution License, which permits unrestricted use, distribution, and reproduction in any medium, provided the original work is properly cited.

\begin{abstract}
Objectives. Substantial studies have demonstrated that an elevated concentration of deoxycholic acid (DCA) in the colonic lumen may play a critical role in the pathogenesis of intestinal barrier dysfunction and inflammatory bowel disease (IBD). The purpose of this study was to investigate the protective effects of GYY4137, as a novel and synthetic $\mathrm{H}_{2} \mathrm{~S}$ donor, on the injury of intestinal barrier induced by sodium deoxycholate (SDC) both in vivo and in vitro. Methods. In this study, Caco-2 monolayers and mouse models with high SDC concentration in the lumen were used to study the effect of GYY4137 on intestinal barrier dysfunction induced by SDC and its underlying mechanisms. Results. In Caco-2 monolayers, a short period of addition of SDC increased the permeability of monolayers obviously, changed distribution of tight junctions (TJs), and improved the phosphorylation level of myosin light chain kinase (MLCK) and myosin light chain (MLC). However, pretreatment with GYY4137 markedly ameliorated the SDC-induced barrier dysfunction. Being injected with GYY4137 could enable mice to resist the SDC-induced injury of the intestinal barrier. Besides, GYY4137 promoted the recovery of the body weight and intestinal barrier histological score of mice with the gavage of SDC. GYY4137 also attenuated the decreased expression level of TJs in mice treated with SDC. Conclusion. Taken together, this research suggests that GYY4137 preserves the intestinal barrier from SDC-induced injury via suppressing the activation of P-MLCK-P-MLC2 signaling pathway and increasing the expression level of tight junctions.
\end{abstract}

\section{Introduction}

The fact that between $1 / 4$ and $1 / 3$ of the Americans can be classified as obese is closely associated with the intake of high-fat diet [1]. Recently, it was shown that high-fat diet impairs intestinal barrier and has correlation with the occurrence of inflammatory bowel disease (IBD) [1-3]. Highfat feeding can increase the concentration and proportion of deoxycholic acid (DCA) in feces, that is the main ingredient of hydrophobic secondary bile acids and whose cytotoxic and intestinal barrier disrupting effects have been fully reported [2-6]. One of the main determinants of intestinal permeability is the intercellular tight junctions (TJs), preventing the translocation of antigens through the epithelium [7]. These antigens play significant roles in the pathogenesis of severe clinical outcomes (e.g., IBD and endotoxemia). Previous studies have reported that DCA or sodium deoxycholate (SDC) could induce altered expression and localization of TJs, leading to the intestinal barrier injury, as well as increased incidence of IBD $[8,9]$.

In recent years, hydrogen sulfide $\left(\mathrm{H}_{2} \mathrm{~S}\right)$, which is traditionally believed to be a toxic gas, has been shown to be a signal molecule, exhibiting a variety of physiological functions which are beneficial to the body [10-12]. Recent st udies reported the potential gastrointestinal protective and ulcer-healing properties of $\mathrm{H}_{2} \mathrm{~S}[12,13]$. In addition, a previous study showed GYY4137 as a $\mathrm{H}_{2} \mathrm{~S}$ donor, that can release low dose of $\mathrm{H}_{2} \mathrm{~S}$ for a long time and that preserves the intestinal barrier function by significantly inhibiting the decreased expression and altered localization of TJs in the context of endotoxemia [14]. However, the effect of GYY4137 on SDC-induced intestinal barrier injury has not been fully elucidated, and the underlying mechanisms have still remained known.

The chronic intake of high-fat feeding can increase the luminal concentration of SDC, which can lead to the 
increased intestinal permeability. In our study, protective effects of GYY4137 on the function of the intestinal barrier in the context of short period and relatively long-term exposure of SDC at the level of cell and animal were elaborated. Our results may exert new insights on potential therapeutic approaches for SDC-related intestinal barrier dysfunction.

\section{Materials and Methods}

2.1. Chemicals and Regents. In our research, SDC, GYY4137, FITC-dextran (4 KDa, FD-4), and FITC-dextran (40 KDa, FD-40) were purchased from Sigma-Aldrich (St. Louis, MO, USA). Primary antibodies for immunofluorescence and western blotting were purchased from companies as follows: Occludin (Thermo Fisher Scientific, Waltham, MA, USA); MLCK (Abcam, Cambridge, UK); P-MLCK (Abcam, Cambridge, UK); ZO-1 (Cell Signaling Technology, Danvers, MA, USA); MLC2 (Cell Signaling Technology, Danvers, MA, USA); and P-MLC2 (Cell Signaling Technology, Danvers, MA, USA).

2.2. Cell Culture. Experiments were conducted with Caco-2 cells which were purchased from American Type Culture Collection (ATCC, Manassas, VA, USA) between passages 28 and 34. Cells were grown at $37^{\circ} \mathrm{C}$ and cultured in Dulbecco's Modified Eagle's Medium (DMEM) supplemented with $4.5 \mathrm{mg} / \mathrm{mL}$ glucose, $10 \%$ fetal bovine serum (FBS), $25 \mathrm{mmol} / \mathrm{L}$ HEPES (4-(2-hydroxyethyl)-1-piperazineethanesulfonic acid), $50 \mathrm{U} / \mathrm{mL}$ penicillin, and $50 \mathrm{U} / \mathrm{mL}$ streptomycin as previously described [14-16]. In order to grow on the transwell, 105 cells with high density were seeded on filters with $0.4 \mu \mathrm{m}$ pore size (Corning Inc., Corning, NY, USA). In the basolateral compartments of transwells, the medium containing various concentrations of GYY4137 was added with or without $2 \mathrm{mM}$ SDC in the apical compartments.

\subsection{Transepithelial Electrical Resistance(TEER) Measurements.} The TEER was measured as described previously [17]. An epithelial volt-ohm meter (ERS-2; Merck Millipore, Burlington, MA, USA) was used to measure the changes of TEER of Caco-2 monolayers. About 21 days after confluence when the epithelial resistance of monolayers reached to $350-550 \Omega \cdot \mathrm{cm}^{2}[18]$, different reagents were added to the transwell as indicated. The TEER was assessed until approximate values were recorded consecutively in three times.

\subsection{Paracellular Marker FITC-Dextran 40 kDa (FD-40) Flux} Measurements. Paracellular permeability was measured using a previous method $[19,20]$. After the treatment described beforehand, monolayers were washed with phosphate-buffered saline (PBS) solution, and then $1 \mathrm{mg} / \mathrm{mL}$ FD4 solution diluted by Hank's balanced salt solution was added to the apical compartments for $2 \mathrm{~h}$. After taking $100 \mu \mathrm{L}$ solution from the basolateral compartments, in $492 \mathrm{~nm}$ excitation and $520 \mathrm{~nm}$ emission filter, the fluorescence of FD-40 flux was assessed with Synergy H2 microplate reader (BioTek Instruments Inc., Winooski, VT, USA). Besides, calibration curves were drafted by serial dilution of FD-40 to determine FD-40 concentrations.

2.5. Western Blot Analysis. A previously reported method was used for the total protein extraction of Caco- 2 monolayers [21]. In vivo, the total protein of the mucosa $(2 \mathrm{~cm})$ in the proximal colon was extracted using a method described previously with some modifications $[14,22]$. The concentration of proteins was measured using bicinchoninic acid (BCA) method (Thermo Fisher Scientific, Waltham, MA, USA). Subsequently, the electrophoresis of extracts involving equivalent quantities of proteins $(25 \mu \mathrm{g})$ was conducted in $6 \%$ or $10 \%$ polyacrylamide gel, and then various proteins were transferred to polyvinylidene difluoride (PVDF) membranes. At room temperature, the membrane was blocked for nonspecific binding for $1 \mathrm{~h}$ (5\% bovine serum albumin (BSA) in TBS-Tween 20 buffer) and then incubated overnight at $4^{\circ} \mathrm{C}$ with primary antibodies $(1: 1000$ dilution). Subsequently, with the corresponding secondary antibodies ( $1: 1000$ dilution), the membrane was incubated at room temperature for $1 \mathrm{~h}$. Finally, blots were developed with electrochemiluminescence (ECL) detection reagents (Merck Millipore, Burlington, MA, USA) and visualized by Syngene GeneGenius gel imaging system (Syngene, Frederick, MD).

2.6. Immunofluorescence of ZO-1 and Occludin in Caco-2 Monolayers. After being treated as indicated previously, cellular distribution of ZO-1 and Occludin was visualized by immunofluorescence as previously reported $[14,21]$. In brief, after being rinsed with PBS, filters were fixed in $100 \%$ methanol overnight at $-20^{\circ} \mathrm{C}$ and $100 \%$ acetone at $-20^{\circ} \mathrm{C}$ for $1 \mathrm{~min}$. Subsequently, at room temperature, filters were blocked with $1 \%$ BSA for $2 \mathrm{~h}$ and then treated with anti-mouse Occludin $(4 \mu \mathrm{g} / \mathrm{ml})$ and anti-rabbit $\mathrm{ZO}-1$ $(6 \mu \mathrm{g} / \mathrm{ml})$ at $4^{\circ} \mathrm{C}$ overnight. After being washing with PBS, filters were incubated with goat anti-mouse IgG conjugated to Alexa555 (Molecular Probes, Eugene, OR, USA) and goat anti-rabbit IgG conjugated to Alexa488 (Molecular Probes, Eugene, OR, USA) in 1\% BSA at room temperature for $1 \mathrm{~h}$ and then rinsed with PBS. In the next step, the Prolong Gold Antifade Reagent (Molecular Probes, Eugene, OR, USA) was used, and cells were stored at $4^{\circ} \mathrm{C}$ in dark until analysis. Under Fluoview 1000 confocal microscope (Olympus, Tokyo, Japan), the fluorescence was visualized.

2.7. Animals. After being purchased from Vital River Inc. (Beijing, China), male C57BL/6 mice (8-weeks old) were raised in the containment unit with access to water and food ad libitum at the Laboratory Animal Center at the Peking University First Hospital (Beijing, China). Before any treatment, the mice had a week to adapt to the environment. The mouse model of SDC with high concentration in the enteric cavity was established by gastric perfusion of SDC (250 mg/kg body weight) to mice once daily for 5 
consecutive days, which maintained from the $1^{\text {st }}$ day to the $5^{\text {th }}$ day. In addition, thirty-six mice were divided into four groups randomly as follows: control; GYY4137 alone; SDC; and SDC+GYY4137. Mice in the control group were injected intraperitoneally with PBS and gavaged with sterile water. Mice in the GYY4137 group were injected intraperitoneally with $50 \mathrm{mg} /(\mathrm{kg} \cdot \mathrm{d}) \mathrm{GYY} 4137$ for 5 consecutive days. Mice in the SDC group were given by gavage of $250 \mathrm{mg} /(\mathrm{kg} \cdot \mathrm{d})$ SDC for consecutive 5 days. Mice in the SDC + GYY4137 group were given by gavage of $250 \mathrm{mg} /$ (kg.d) SDC and injected intraperitoneally with $50 \mathrm{mg} /(\mathrm{kg} \cdot \mathrm{d})$ GYY4137 for 5 consecutive days. Body weight was daily recorded until the $5^{\text {th }}$ day. All mice were euthanized $12 \mathrm{~h}$ after final administration. All procedures of this study were approved by the Institutional Review Board of Peking University First Hospital.

2.8. Measurement of Intestinal Permeability in Mice. The epithelial barrier permeability of mice was evaluated by assessing the concentration of FD-4 in plasma as previously described [23]. Briefly, $12 \mathrm{~h}$ after final administration, mice were gavaged with FD-4 $(60 \mathrm{mg} / 100 \mathrm{~g}$ body weight). Blood was collected $4 \mathrm{~h}$ after FD-4 gavage by cardiac puncture in EDTA-coated tubes. Subsequently, the plasma was collected by centrifuging at $3000 \mathrm{rpm}$ for $15 \mathrm{~min}$ at $4^{\circ} \mathrm{C}$, and its fluorescent signal of plasma was assessed with a Synergy $\mathrm{H} 2$ microplate reader (BioTek Instruments Inc., Winooski, VT, USA) using $492 \mathrm{~nm}$ excitation and $520 \mathrm{~nm}$ emission filters. Standard curves were generated by serial dilution of FD-40 to determine FD-40 concentrations.

2.9. Histological Assessment. The proximal colons (3 per each group) collected from mice in different groups were excised and embedded in paraffin. Sections (thickness, $4 \mu \mathrm{m}$ ) were cut and stained with hematoxylin and eosin (H\&E). Images were observed using a Zeiss Image light microscope (magnification: 20x; Carl Zeiss AG, Oberkochen, Germany). The degree of histopathologic changes was graded as previously described: Score 0, normal histological findings. Score 1, mucosa: villus blunting, loss of crypt architecture, sparse inflammatory cell infiltration, vacuolization, and edema; muscle layer: normal. Score 2, mucosa: villus blunting with fattened and vacuolated cells, crypt necrosis, intense inflammatory cell infiltration, vacuolization, and edema; muscle layer: normal. Score 3, mucosa: villus blunting with fattened and vacuolated cells, crypt necrosis, intense inflammatory cell infiltration, vacuolization, and edema [24].

2.10. Statistical Analysis. The results were expressed as mean \pm standard error of the mean (SEM) and analyzed using a Student's $t$-test for unpaired data and one-way analysis of variance (ANOVA) to compare groups whenever required by GraphPad Prism 5.0 software. A $P$ value $<0.05$ was considered to be statistically significant.

\section{Results}

3.1. The Effects of SDC on the TEER of Caco-2 Monolayers. We assessed the barrier disruption by a reduction in the TEER after the application of SDC in the Caco-2 monolayer. We tested different concentrations (0-2.4 mM) of SDC and found that $2 \mathrm{mM}$ concentration or greater was sufficient to significantly decrease the TEER $(P<0.05$, Figure $1(\mathrm{a}))$. In a relatively short time, $30 \mathrm{~min}$, the TEER declined rapidly to under $50 \%$ of its initial level. At the end of $30 \mathrm{~min}$, the downtown of TEER induced by SDC was pronounced enough in minimum dose. The TEER did not show further remarkable drop over time (Figure 1(a)).

We want to find a minimum concentration of SDC, which could cause a $>50 \%$ fall in the TEER of Caco-2 monolayer at $30 \mathrm{~min}$. After adding various concentrations of $\mathrm{SDC}$ to the apical compartments for $30 \mathrm{~min}$, the concentration of $2 \mathrm{mM}$ would be used in the subsequent experiments (Figure 1(b)).

3.2. The Protective Effects of GYY4137 on SDC-Induced Injuries of Intestinal Barrier. TEER and FD-40 flux were used to assess the monolayer barrier function. After being exposed to $2 \mathrm{mM}$ SDC for $30 \mathrm{~min}$, TEER decreased $(P<0.05$, Figure 1(c)), while FD-40 flux significantly increased $(P<0.05$, Figure $1(\mathrm{~d}))$. In order to explore the protective effects of GYY4137 on SDC-induced injuries of barrier function, the monolayers were pretreated with increasing doses of GYY4137 (50, 100, and $200 \mu \mathrm{M})$ for $48 \mathrm{~h}$ followed by being exposed to $2 \mathrm{mM}$ SDC for $30 \mathrm{~min}$. Preincubation with different concentrations of GYY4137 could in a dose-dependent manner notably alleviate the fall of TEER and the increase of FD-40 flux induced by SDC (Figures 1(c) and $1(\mathrm{~d}))$. GYY4137, at $200 \mu \mathrm{M}$ concentration, produced the most significant effect, and thereafter, $200 \mu \mathrm{M}$ GYY4137 was used in the following experiments.

3.3. The Effects of GYY4137 and SDC on Tight Junctions. The decrease of expression level of TJs and altered localization of TJs can both cause injury to the intestinal epithelial barrier function [14]. However, GYY4137 or exposure of SDC for $30 \mathrm{~min}$ could not exert significant influence on the expression level of TJs (Figure 2). Thus, immunofluorescences of ZO-1 and Occludin were assessed to examine whether SDC could change the permeability of monolayer by altering the distribution of TJs.

In general, smooth edges and typical "chicken wire" were normal shapes of immunofluorescences of ZO-1 and Occludin [14]. However, after being added with $2 \mathrm{mM}$ SDC for $30 \mathrm{~min}$, the cellular edges became serrated. Network patterns of ZO-1 and Occludin were disrupted with areas of discontinuous and irregular labeling intensity. These results indicated that SDC caused abnormal localization of the TJs. However, pretreatment with GYY4137 attenuated the changes caused by SDC exposure. With preincubation of $200 \mu \mathrm{M}$ GYY4137 for $48 \mathrm{~h}, \mathrm{ZO}-1$ and Occludin remained in a "chicken wire" shape after adding SDC to the Caco-2 monolayer (Figure 3). 


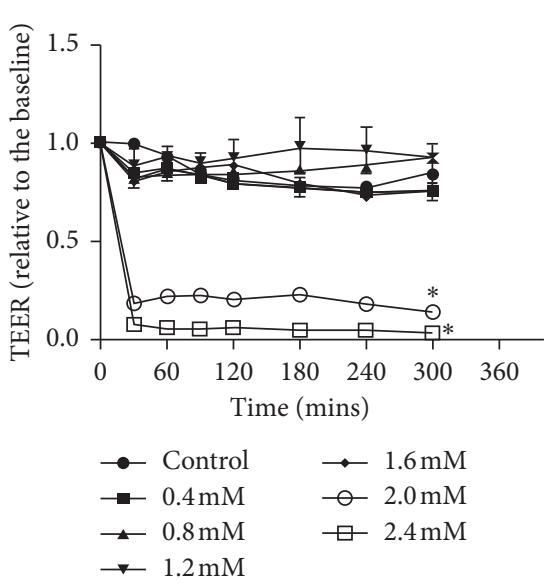

(a)

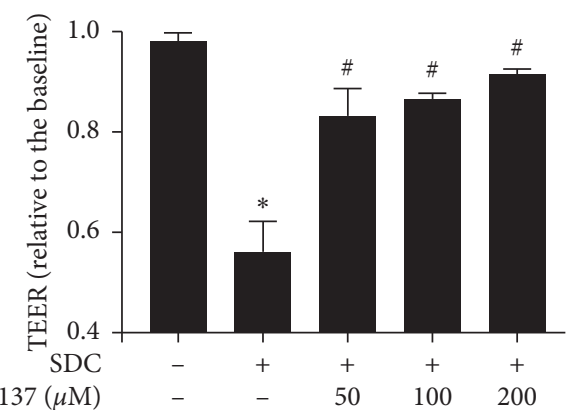

(c)

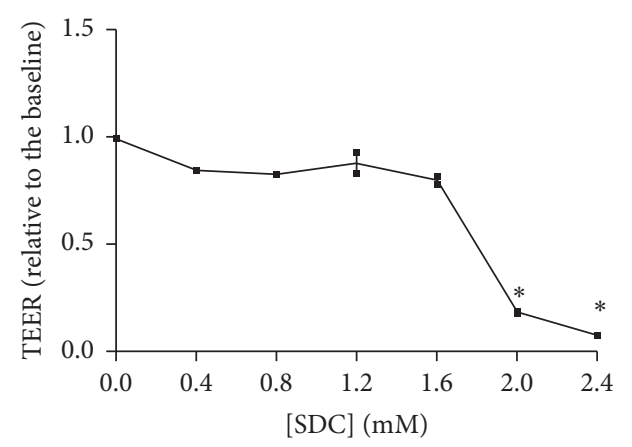

(b)

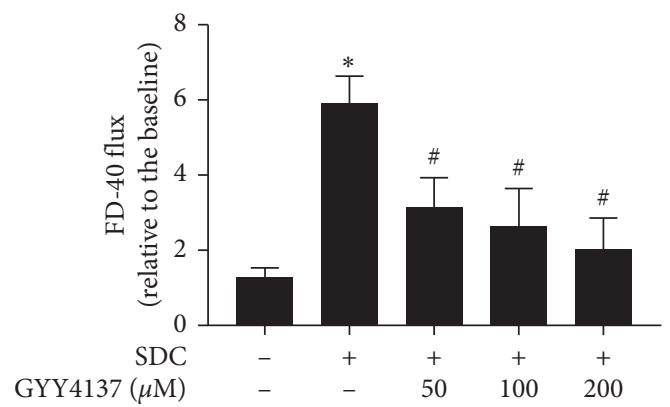

(d)

FIGURE 1: Destructive effects of SDC on Caco-2 monolayer barrier function and GYY4137 ameliorates intestinal epithelial barrier dysfunction induced by SDC. (a) Caco-2 monolayers were treated with different concentrations $(0-2.4 \mathrm{mM})$ of SDC. $2 \mathrm{Mm}$ or greater concentration could significantly decrease the TEER at $30 \mathrm{~min}$. (b) Dose-response of the TEER of Caco-2 monolayers treated with different concentrations of SDC for $30 \mathrm{~min}$. Concentration $\geq 2 \mathrm{mM}$ significantly degraded the TEER. (c) Caco-2 monolayers were preincubated with or without 50,100 , and $200 \mu \mathrm{M}$ GYY 4137 for $48 \mathrm{~h}$ and then treated in the presence or absence of $2 \mathrm{mM}$ SDC for $30 \mathrm{~min}$. GYY4137 significantly attenuated TEER reduction induced by SDC treatment. (d) Caco-2 monolayers were treated as described. The increase of FD-40 flux induced by SDC was significantly attenuated by GYY4137 treatment. Results were expressed as mean \pm SEM $(n=3)$. ${ }^{*} P<0.05$ vs. control.

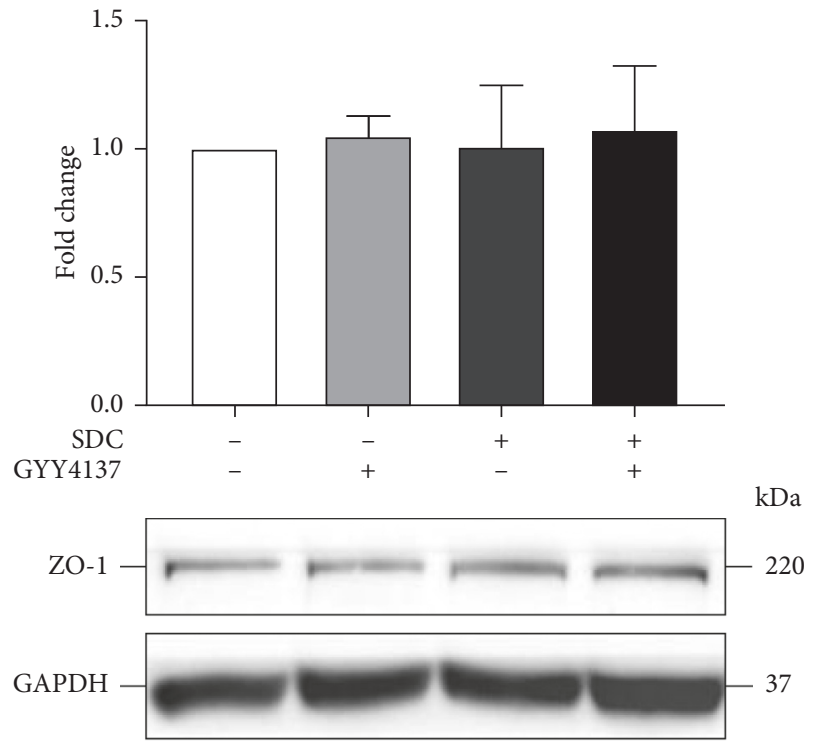

(a)

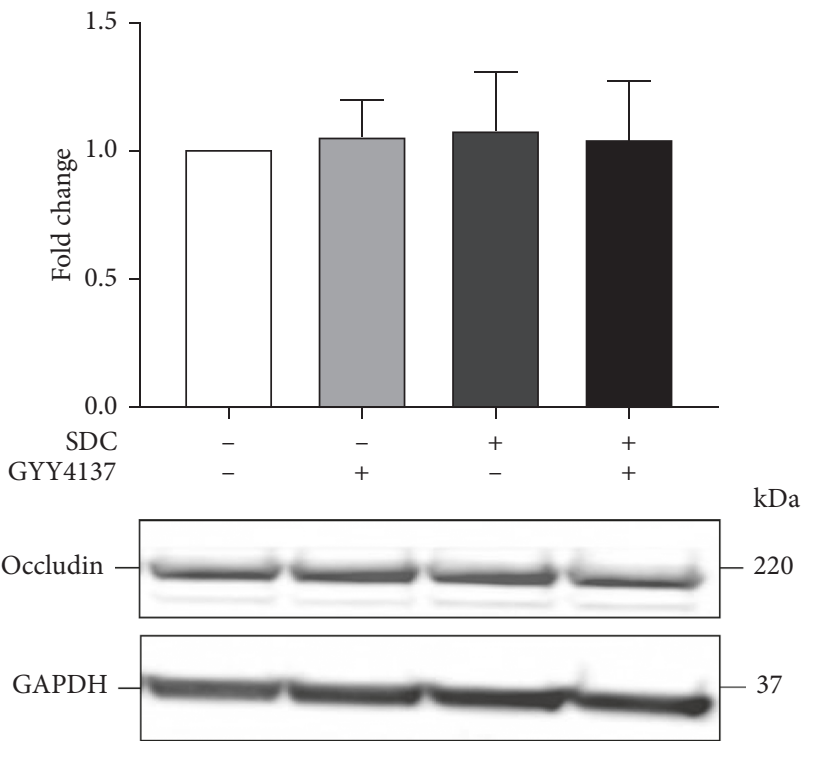

(b)

FIgURE 2: The effects of GYY4137 and SDC on the expression level of TJs in monolayers. Caco-2 monolayers were preincubated with or without $200 \mu \mathrm{M}$ GYY4137 for $48 \mathrm{~h}$ and then treated in the presence or absence of $2 \mathrm{mM}$ SDC for $30 \mathrm{~min}$. The total protein of monolayers was harvested after treatment for western blot assay. Results were expressed as mean $\pm \operatorname{SEM}(n=3)$. 

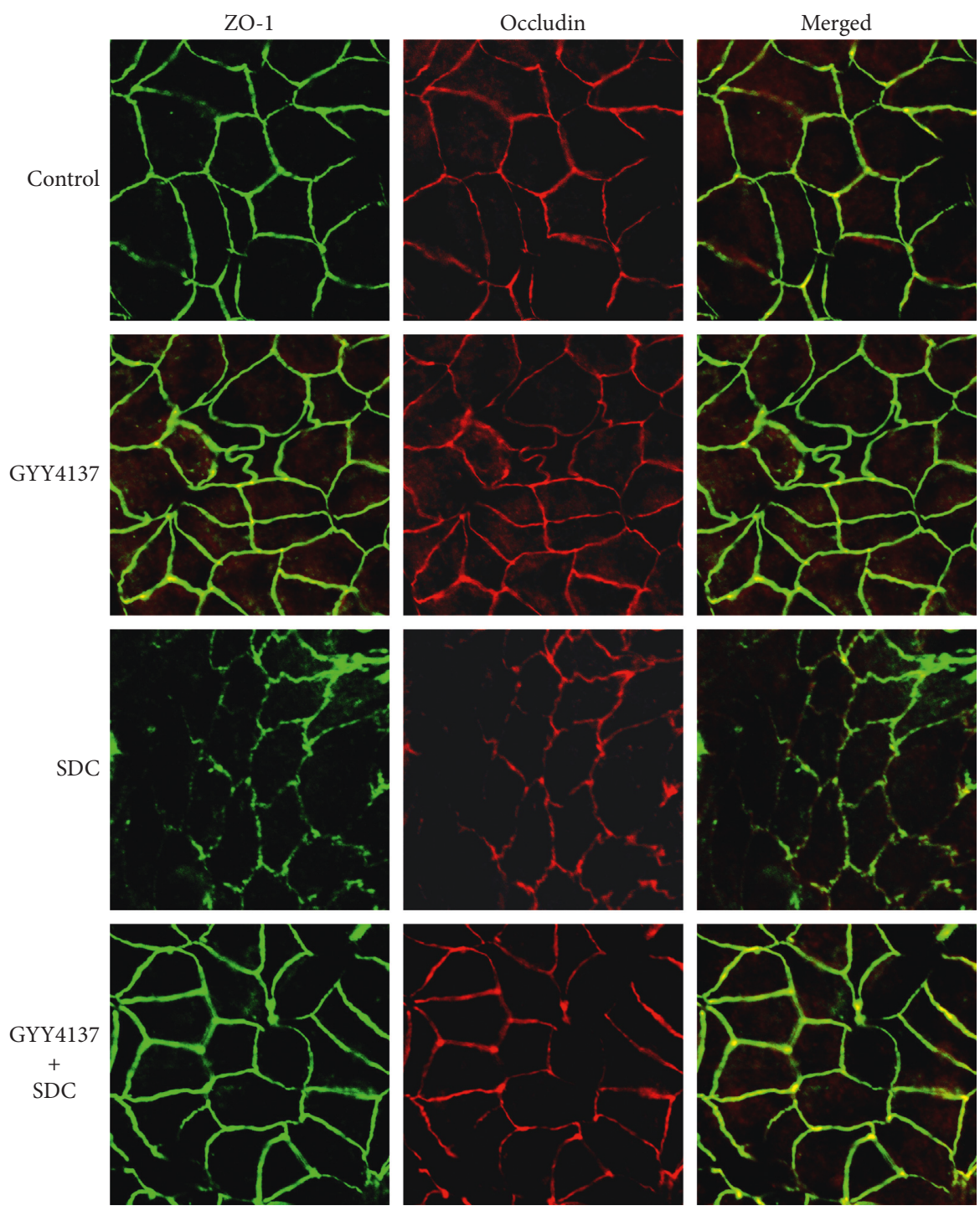

FIGURE 3: The effects of GYY4137 and SDC on the localization of TJs in monolayers. ZO-1 and Occludin were stained by immunofluorescence. The altered localization of TJs induced by SDC was ameliorated by GYY4137.

3.4. The Effects of GYY4137 on SDC-Induced Activation of $P$-MLCK-P-MLC2 Signaling Pathway. Previous studies have reported the pivotal role of MLCK-P-MLC2 pathway in the physiological and pathological regulation of the localization of TJs $[14,25]$. However, GYY4137 or treatment of SDC for $30 \mathrm{~min}$, that is a relatively short time, had no significant influence on the expression level of MLCK (Figure 4(a)). On the other hand, addition of SDC transiently increased MLCK phosphorylation (Figure 4(b)), as the active form of MLCK and a key regulator of tight junction distribution which could increase the phosphorylation level of MLC2 [26, 27].

After the treatment of Caco-2 monolayers with SDC for $30 \mathrm{~min}$, there was an obvious increase in the phosphorylation level of MLCK and MLC2 compared with control $(P<0.05)$. However, compared with Caco-2 monolayers treated with SDC, pretreatment with GYY4137 for $48 \mathrm{~h}$ significantly inhibited the increase of phosphorylation level of MLCK and MLC2 $(P<0.05$, Figures $4(\mathrm{~b})$ and $4(\mathrm{c}))$. This result showed that GYY4137 suppressed P-MLCK-P-MLC2 signaling pathway and then ameliorated SDC-induced intestinal barrier destruction.

3.5. GYY4137 Ameliorates SDC-Induced Weight Loss of Mice. Body weight sharply decreased due to SDC treatment, in contrast to control and GYY4137 groups (Figure 5(a)). The weight loss due to SDC treatment was significantly improved in mice administrated with GYY4137 on the 5th day $(P<0.05$, Figure 5(a)).

3.6. GYY4137 Had Protective Effects on the Intestinal Barrier Function in Mice Treated with SDC. In mice treated with SDC, the FD- 4 concentration in the plasma was significantly higher than that in the control $(P<0.05$, Figure 5(b)). However, the concentration of FD-4 was markedly decreased by cotreatment of GYY4137 $(P<0.05$, Figure 5(b)). These results suggested that GYY4137 attenuated the injuries induced by SDC on intestinal barrier function. 


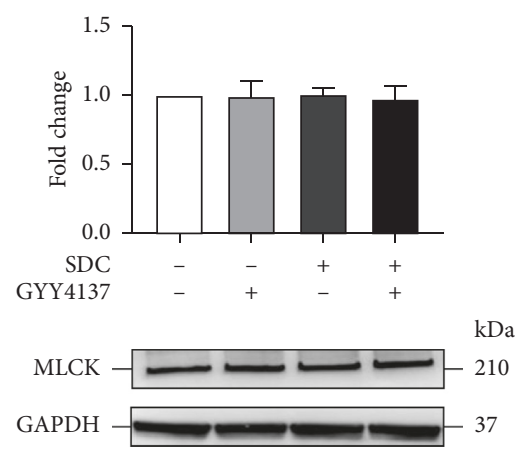

(a)
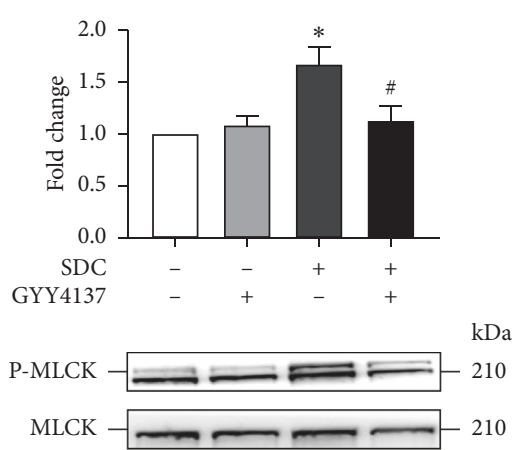

(b)

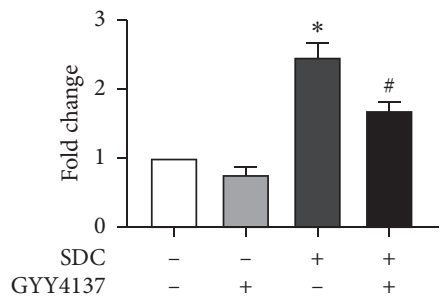

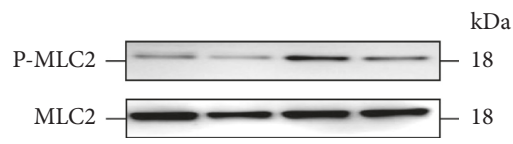

(c)

FIgURE 4: The effects of GYY4137 on the status of P-MLCK-P-MLC2 signaling pathway in Caco-2 monolayers with short-term SDC treatment. Caco-2 monolayers were treated as described in Figure 3. (a-c) GYY4137 inhibited SDC-induced increased phosphorylation of MLCK and MLC2. Results were expressed as mean \pm SEM $(n=3) .{ }^{*} P<0.05$ vs. control. ${ }^{\#} P<0.05$ vs. SDC.

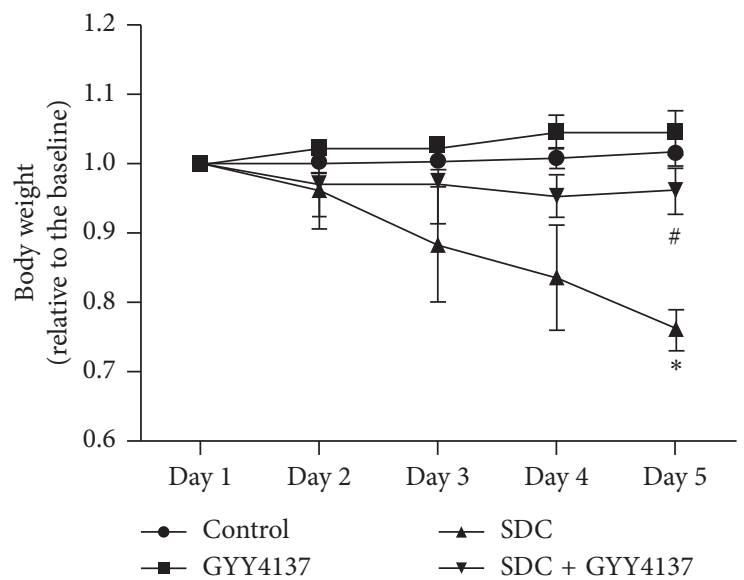

(a)

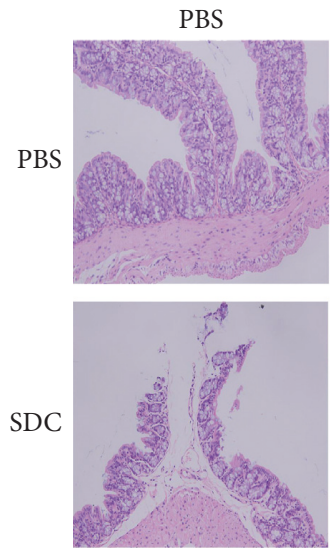

(c)

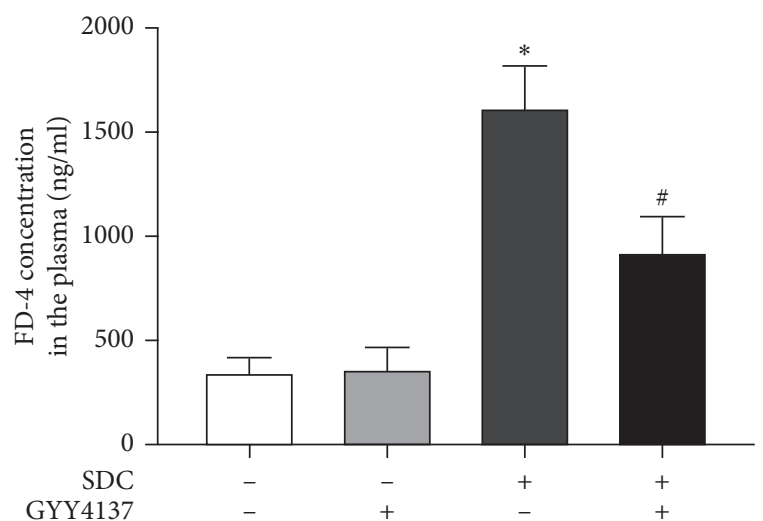

(b)

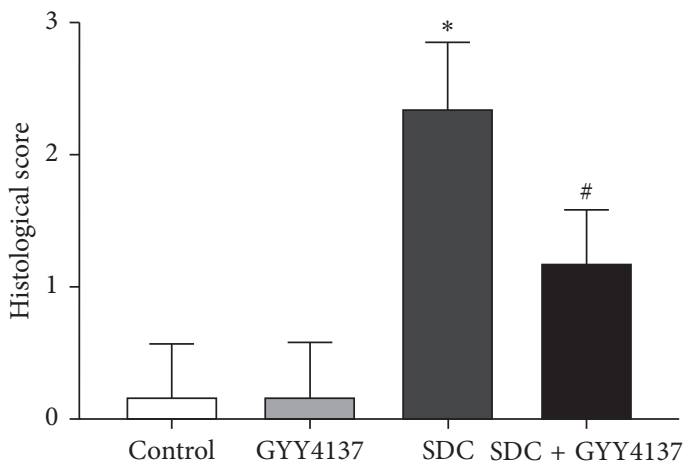

(d)

FIGURE 5: The effects of GYY4137 on the body weight, barrier function, and histological score of mice with the gavage of SDC. (a) GYY4137 obviously inhibited the decreased body weight of mice treated with SDC. (b) GYY4137 remarkably ameliorated the broken intestinal barrier featured by increased FD-4 flux in mice treated with SDC. (c) GYY4137 attenuated the mucosal damage caused by SDC. (d) GYY4137 significantly attenuated the increased histological score in mice with the gavage of SDC. All experiments were performed using 6 mice per experimental group and repeated at least three times. Results were expressed as mean $\pm \operatorname{SEM}(n=6) .{ }^{*} P<0.05$ vs. control. ${ }^{\#} P<0.05$ vs. SDC.

3.7. GYY4137 Improved the Histological Status in Mice Treated with SDC. Cotreatment with GYY4137 attenuated the histological damage of colon epithelium in mice with
SDC treatment, featured by villus stunting, deciduous epithelial cells, crypt disruption, and discrete submucosal (Figures 5(c) and 5(d)). 

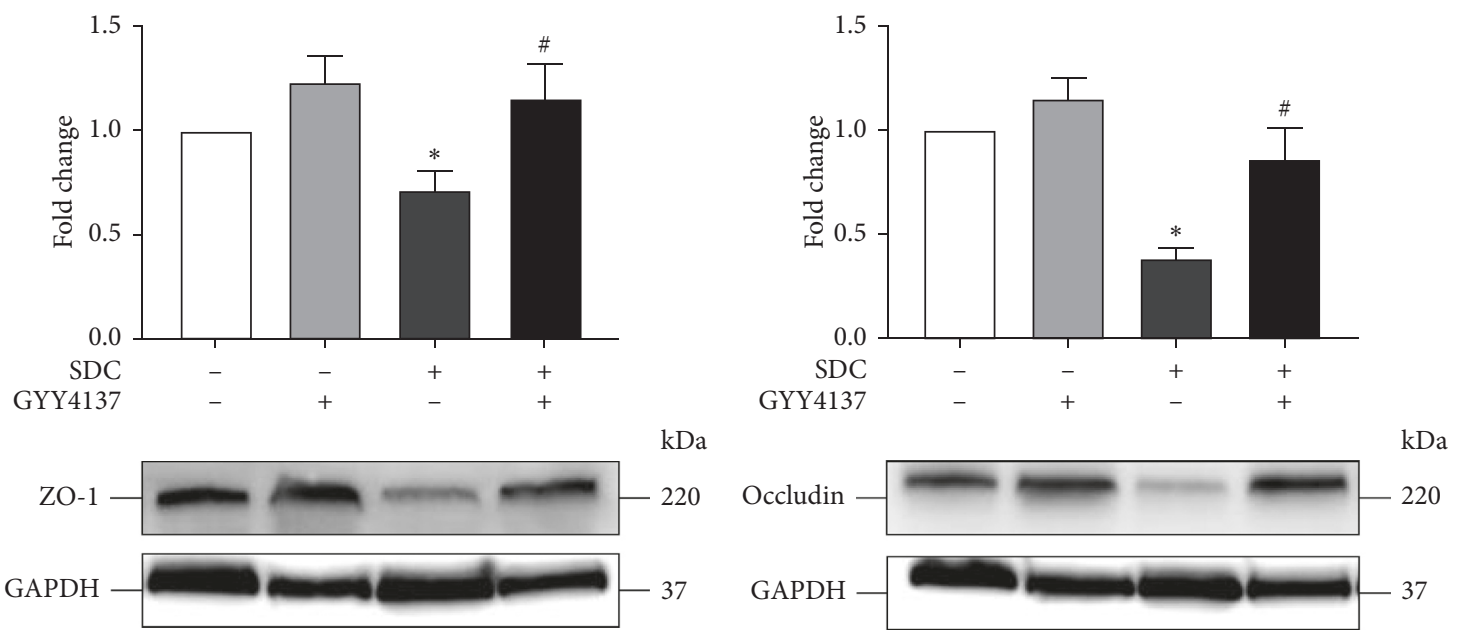

(a)

(b)

FIgURE 6: The effects of GYY4137 on the expression level of TJs of mice with the gavage of SDC. GYY4137 significantly inhibited the decreased expression of ZO-1 and Occludin in the proximal colon of mice with the gavage of SDC. All experiments were performed using 6 mice per experimental group and repeated at least three times. Results were expressed as mean $\pm \operatorname{SEM}(n=6) .{ }^{*} P<0.05$ vs. control. ${ }^{\#} P<0.05$ vs. SDC.

3.8. GYY4137 Inhibited SDC-Induced Decreased Expression of TJs in Mice. The treatment by SDC for a relatively short time, $30 \mathrm{~min}$, could not affect the expression level of TJs. However, in mice, exposure to SDC for a relatively long time, 5 days, significantly decreased the expression level of TJs. In the proximal colon, GYY4137 notably ameliorated the reduced expression level of TJs $(P<0.05$, Figure 6).

\section{Discussion}

TJs, whose expression level and localization can determine intestinal barrier function, are an essential component of intestinal mucosal mechanical barrier $[7,14]$. A large body of evidence suggests that intestinal barrier dysfunction plays a pivotal role in the pathogenesis of multiple enteropathies (e.g., IBD). People with long-term high-fat feeding have higher rates of IBD [1-3] and intraluminal concentrations of hydrophobic secondary bile acids $[2,3]$. The high concentration of DCA, the main secondary bile acid compromising approximately $20 \%$ of bile acids [28, 29], can damage the function of intestinal barrier via altering expression [30] and localization $[28,31]$ of TJs. Thus, it is a prospective research to seek potential therapeutic reagents for individuals with high concentrations of bile acids in the lumen who may have long-term high-fat diet.

In this study, we used Caco-2 monolayers and the effects of SDC on the intestinal barrier function were investigated. Previous studies have reported that SDC can break the barrier function of monolayers in a relatively short period, in about $1 \mathrm{~h}[28,32]$, in which our results were consistent with previous studies. Our research indicated that $2 \mathrm{mM}$ concentration of SDC or greater was sufficient to decrease the TEER to $<50 \%$ of its initial level at $30 \mathrm{~min}$. Previous studies indicated that SDC can damage the epithelial cells by direct destructive effects and inducing apoptosis $[33,34]$. To ensure the highest activity of cells, we selected a relatively short processing time and a low concentration, $30 \mathrm{~min}$ and $2 \mathrm{mM}$, in the subsequent experiments. Previous research has shown that bile acids exist in the human intestine at a concentration with a typical range from 2-6 $\mathrm{mM}$ [28]; therefore, doses of SDC used in this study fall within the physiologic range. Our study suggested that expose of Caco- 2 monolayers to $2 \mathrm{mM}$ SDC for $30 \mathrm{~min}$ did not affect the expression level of TJs. The immunofluorescence suggested that the principal factor in SDC-induced destruction of intestinal barrier at $30 \mathrm{~min}$ was the altered localization of TJs.

GYY4137, as a novel and ideal H2S donor, is stable in vitro and in vivo. Compared with $\mathrm{NaHS}$, in longer term, GYY4137 can release $\mathrm{H}_{2} \mathrm{~S}$ stably in the physiological context $[14,35] . \mathrm{H}_{2} \mathrm{~S}$, as a new type of gaseous signal molecule, has anti-inflammatory and antiapoptosis influences on multiple tissues $[36,37]$. However, the influences of GYY4137 on the SDC-induced injury of barrier function and the underlying mechanisms need to be elucidated. A previous research revealed that GYY4137 could ameliorate intestinal barrier injury by regulating the expression level and localization of TJs in the context of endotoxemia [14]. In the present study, by in vitro utilizing Caco-2 monolayers, we realized that the pretreatment with GYY4137 for $48 \mathrm{~h}$ significantly attenuated the SDC-induced decrease of TEER and increase of FD-40 flux. GYY4137 also was able to ameliorate the altered localization of TJs caused by SDC.

Additionally, MLCK-P-MLC2 is a very classic signaling pathway to regulate barrier function by altering the distribution of TJs. Actin-myosin filaments are able to contract when the phosphorylation level of MLC2 increase, resulting in rapidly altered distribution of TJs and broken intestinal barrier [22, 25]. In our study, the findings of immunoblotting revealed that SDC notably upregulated the phosphorylation of MLC2. However, $30 \mathrm{~min}$, SDC was unable to regulate expression level of MLCK. MLCK phosphorylation, a key regulator of the localization of TJs, representing the 
active form of MLCK, can increase phosphorylation of MLC2 [26, 27]. Moreover, SDC can enhance the phosphorylation level of MLCK, manifesting the involvement of P-MLCK-P-MLC2 pathway in SDC-caused barrier dysfunction in Caco-2 monolayers. Subsequently, we assessed the effects of GYY4137 on SDC-induced activation of P-MLCK-P-MLC2 signaling pathway. The findings showed that pretreatment with GYY4137 remarkably suppressed the activation of P-MLCK-P-MLC2 signaling pathway induced by SDC.

In order to further verify our results, we established a mouse model with high SDC concentration in the lumen. It was disclosed that GYY4137 could significantly suppress the sharply decreased body weight, as one of the representative symptoms of broken intestinal barrier function, which was induced by SDC treatment. The permeability of intestinal barrier was also examined by assessing the plasma concentration of FD-4. The results showed that GYY4137 was able to protect the mice from the injuries of intestinal barrier caused by SDC. Histological assessment of the colon epithelium suggested that GYY4137 could promote the recovery of abnormal histopathological features induced by SDC. In addition, GYY4137 alleviated the decreased expression level of TJs caused by SDC.

In a relatively short time in vitro, SDC was able to break barrier function by regulating the localization of TJs. However, in a relatively long time in vivo, SDC exerted destructive effect on the intestinal barrier by decreasing the expression level of TJs. Furthermore, GYY4137 can affect both localization and expression level of TJs to exert protective effects on the intestinal barrier.

In summary, the present study illustrated that GYY4137 could ameliorate SDC-induced injuries of intestinal barrier both in vitro and in vivo, and the suppression of SDC-induced activation of P-MLCK-P-MLC2 signaling pathway and decreased expression level of TJs might be one of the underlying mechanisms of protective effects. Our research may provide a reliable basis for the application of GYY4137 against bile acids-related gut leakiness, and this study has also a great potential to become a novel therapeutic approach to prevent and remedy high-fat diet-related IBD.

\section{Data Availability}

All data generated or analysed during this study are available from the corresponding author on reasonable request.

\section{Conflicts of Interest}

The authors declare that there are no conflicts of interest.

\section{Authors' Contributions}

Zeyang Chen and Jianqiang Tang contributed equally to this work.

\section{Acknowledgments}

We thank Doctor Yang Jiao for the excellent technical assistance in our animal experiments. This work was supported by the National Natural Science Foundation of China (no. 8177030343).

\section{References}

[1] T. Suzuki and H. Hara, "Dietary fat and bile juice, but not obesity, are responsible for the increase in small intestinal permeability induced through the suppression of tight junction protein expression in LETO and OLETF rats," Nutrition \& Metabolism, vol. 7, no. 1, p. 19, 2010.

[2] L. K. Stenman, R. Holma, and R. Korpela, "High-fat-induced intestinal permeability dysfunction associated with altered fecal bile acids," World Journal of Gastroenterology, vol. 18, no. 9, pp. 923-929, 2012.

[3] L. K. Stenman, R. Holma, A. Eggert, and R. Korpela, "A novel mechanism for gut barrier dysfunction by dietary fat: epithelial disruption by hydrophobic bile acids," American Journal of Physiology-Gastrointestinal and Liver Physiology, vol. 304, no. 3, pp. G227-G234, 2013.

[4] A. Münch, M. Ström, and J. D. Söderholm, "Dihydroxy bile acids increase mucosal permeability and bacterial uptake in human colon biopsies," Scandinavian Journal of Gastroenterology, vol. 42, no. 10, pp. 1167-1174, 2007.

[5] K. J. Goerg, M. Gross, G. Nell, W. Rummel, and L. Schulz, "Comparative study of the effect of cholera toxin and sodium deoxycholate on the paracellular permeability and on net fluid and electrolyte transfer in the rat colon," Naunyn-Schmiedeberg's Archives of Pharmacology, vol. 312, no. 1, pp. 91-97, 1980.

[6] C. K. Henrikson, R. A. Argenzio, J. A. Liacos, and J. Khosla, "Morphologic and functional effects of bile salt on the porcine colon during injury and repair," Laboratory Investigation; a Journal of Technical Methods and Pathology, vol. 60, no. 1, pp. 72-87, 1989.

[7] D. C. Baumgart and A. U. Dignass, "Intestinal barrier function," Current Opinion in Clinical Nutrition and Metabolic Care, vol. 5, no. 6, pp. 685-694, 2002.

[8] Y. Araki, T. Katoh, A. Ogawa et al., "Bile acid modulates transepithelial permeability via the generation of reactive oxygen species in the Caco-2 cell line," Free Radical Biology and Medicine, vol. 39, no. 6, pp. 769-780, 2005.

[9] F. Raimondi, P. Santoro, M. V. Barone et al., "Bile acids modulate tight junction structure and barrier function of Caco-2 monolayers via EGFR activation," American Journal of Physiology-Gastrointestinal and Liver Physiology, vol. 294, no. 4, pp. G906-G913, 2008.

[10] Y. Mikami, N. Shibuya, Y. Kimura, N. Nagahara, M. Yamada, and H. Kimura, "Hydrogen sulfide protects the retina from light-induced degeneration by the modulation of $\mathrm{Ca}^{2+}$ Influx," Journal of Biological Chemistry, vol. 286, no. 45, pp. 3937939386, 2011.

[11] Z. Fu, X. Liu, B. Geng, L. Fang, and C. Tang, "Hydrogen sulfide protects rat lung from ischemia-reperfusion injury," Life Sciences, vol. 82, no. 23-24, pp. 1196-1202, 2008.

[12] J. L. Wallace, L. Vong, W. McKnight, M. Dicay, and G. R. Martin, "Endogenous and exogenous hydrogen sulfide promotes resolution of colitis in rats," Gastroenterology, vol. 137, no. 2, pp. 569-578, 2009.

[13] M. Magierowski, K. Magierowska, S. Kwiecien, and T. Brzozowski, "Gaseous mediators nitric oxide and hydrogen sulfide in the mechanism of gastrointestinal integrity, protection and ulcer healing," Molecules: A Journal of Synthetic Chemistry and Natural Product Chemistry, vol. 20, no. 5, pp. 9099-9123, 2015. 
[14] S. Chen, D. Bu, Y. Ma et al., "GYY4137 ameliorates intestinal barrier injury in a mouse model of endotoxemia," Biochemical Pharmacology, vol. 118, pp. 59-67, 2016.

[15] D. Ye, S. Guo, R. Al-Sadi, and T. Y. Ma, "MicroRNA regulation of intestinal epithelial tight junction permeability," Gastroenterology, vol. 141, no. 4, pp. 1323-1333, 2011.

[16] L. F. Xu, X. Teng, J. Guo, and M. Sun, "Protective effect of intestinal trefoil factor on injury of intestinal epithelial tight junction induced by platelet activating factor," Inflammation, vol. 35, no. 1, pp. 308-315, 2012.

[17] T. Y. Ma, N. T. Hoa, D. D. Tran et al., "Cytochalasin B modulation of Caco-2 tight junction barrier: role of myosin light chain kinase," American Journal of Physiology. Gastrointestinal and Liver Physiology, vol. 279, no. 5, pp. G875G885, 2000.

[18] D. Qiu, G. Zhao, Y. Aoki et al., "Immunosuppressant PG490 (triptolide) inhibits T-cell interleukin-2 expression at the level of purine-box/nuclear factor of activated T-cells and NFkappaB transcriptional activation," The Journal of Biological Chemistry, vol. 274, no. 19, pp. 13443-13450, 1999.

[19] N. Schlegel, M. Meir, V. Spindler, C. T. Germer, and J. Waschke, "Differential role of Rho GTPases in intestinal epithelial barrier regulation in vitro," Journal of Cellular Physiology, vol. 226, no. 5, pp. 1196-1203, 2011.

[20] M. Schliwa, "Action of cytochalasin D on cytoskeletal networks," The Journal of Cell Biology, vol. 92, no. 1, pp. 79-91, 1982.

[21] T. Y. Ma, G. K. Iwamoto, N. T. Hoa et al., “TNF-alpha-induced increase in intestinal epithelial tight junction permeability requires NF-kappa B activation," American Journal of Physiology. Gastrointestinal and Liver Physiology, vol. 286, no. 3, pp. G367-G376, 2004.

[22] R. Moriez, C. Salvador-Cartier, V. Theodorou, J. Fioramonti, H. Eutamene, and L. Bueno, "Myosin light chain kinase is involved in lipopolysaccharide-induced disruption of colonic epithelial barrier and bacterial translocation in rats," The American Journal of Pathology, vol. 167, no. 4, pp. 1071-1079, 2005.

[23] H. Zhao, H. Zhang, H. Wu et al., "Protective role of 1,25(OH) 2 vitamin D3 in the mucosal injury and epithelial barrier disruption in DSS-induced acute colitis in mice," $B M C$ Gastroenterology, vol. 12, no. 1, p. 57, 2012.

[24] Y. Wang, L. Sun, S. Chen et al., "The administration of Escherichia coli Nissle 1917 ameliorates irinotecan-induced intestinal barrier dysfunction and gut microbial dysbiosis in mice," Life Sciences, vol. 231, Article ID 116529, 2019.

[25] L. Shen, E. D. Black, E. D. Witkowski et al., "Myosin light chain phosphorylation regulates barrier function by remodeling tight junction structure," Journal of Cell Science, vol. 119, no. 10, pp. 2095-2106, 2006.

[26] K. E. Cunningham and J. R. Turner, "Myosin light chain kinase: pulling the strings of epithelial tight junction function," Annals of the New York Academy of Sciences, vol. 1258, no. 1, pp. 34-42, 2012.

[27] A. Boire, Y. Zou, J. Shieh, D. G. Macalinao, E. Pentsova, and J. Massagué, "Complement component 3 adapts the cerebrospinal fluid for leptomeningeal metastasis," Cell, vol. 168, no. 6, pp. 1101-1113, 2017.

[28] A. Edelstein, D. Fink, M. Musch et al., "Protective effects of nonionic triblock copolymers on bile acid-mediated epithelial barrier disruption," Shock, vol. 36, no. 5, pp. 451-457, 2011.

[29] S. S. Rossi, J. L. Converse, and A. F. Hofmann, "High pressure liquid chromatographic analysis of conjugated bile acids in human bile: simultaneous resolution of sulfated and unsulfated lithocholyl amidates and the common conjugated bile acids," Journal of Lipid Research, vol. 28, no. 5, pp. 589-595, 1987.

[30] Y. Murakami, S. Tanabe, and T. Suzuki, "High-fat diet-induced intestinal hyperpermeability is associated with increased bile acids in the large intestine of mice," Journal of Food Science, vol. 81, no. 1, pp. H216-H222, 2016.

[31] Y. Sun, B. M. Fihn, H. Sjövall, and M. Jodal, "Enteric neurones modulate the colonic permeability response to luminal bile acids in rat colon in vivo," Gut, vol. 53, no. 3, pp. 362-367, 2004.

[32] Y. Tan, J. Qi, Y. Lu, F. Hu, Z. Yin, and W. Wu, "Lecithin in mixed micelles attenuates the cytotoxicity of bile salts in Caco2 cells," Toxicology in Vitro: An International Journal Published in Association with BIBRA, vol. 27, no. 2, pp. 714-720, 2013.

[33] C. J. O’Connor, R. G. Wallace, K. Iwamoto, T. Taguchi, and J. Sunamoto, "Bile salt damage of egg phosphatidylcholine liposomes," Biochimica et biophysica acta, vol. 817, no. 1, pp. 95-102, 1985.

[34] K. M. McCarty, T. J. Smith, W. Zhou et al., "Polymorphisms in XPD (Asp312Asn and Lys751Gln) genes, sunburn and arsenic-related skin lesions," Carcinogenesis, vol. 28, no. 8, pp. 1697-1702, 2007.

[35] P. Rose, B. W. Dymock, and P. K. Moore, "GYY4137, a novel water-soluble, H2S-releasing molecule," Methods in Enzymology, vol. 554, pp. 143-167, 2015.

[36] R. C. Zanardo, V. Brancaleone, E. Distrutti, S. Fiorucci, G. Cirino, and J. L. Wallace, "Hydrogen sulfide is an endogenous modulator of leukocyte-mediated inflammation," FASEB Journal, vol. 20, no. 12, pp. 2118-2120, 2006.

[37] G. Meng, J. Wang, Y. Xiao et al., "GYY4137 protects against myocardial ischemia and reperfusion injury by attenuating oxidative stress and apoptosis in rats," Journal of Biomedical Research, vol. 29, no. 3, pp. 203-213, 2015. 


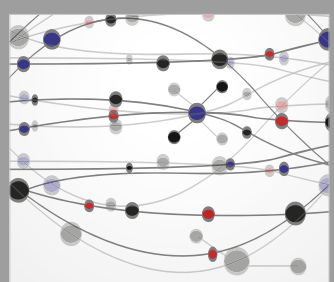

The Scientific World Journal
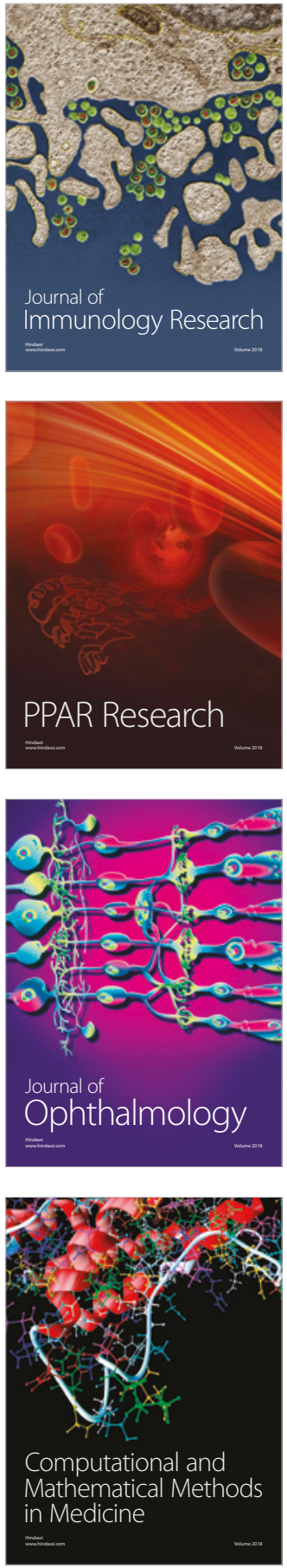

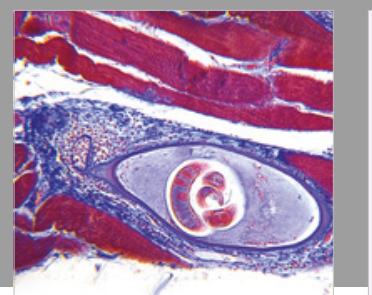

Gastroenterology Research and Practice

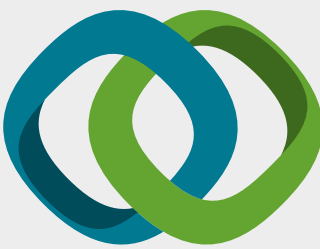

\section{Hindawi}

Submit your manuscripts at

www.hindawi.com
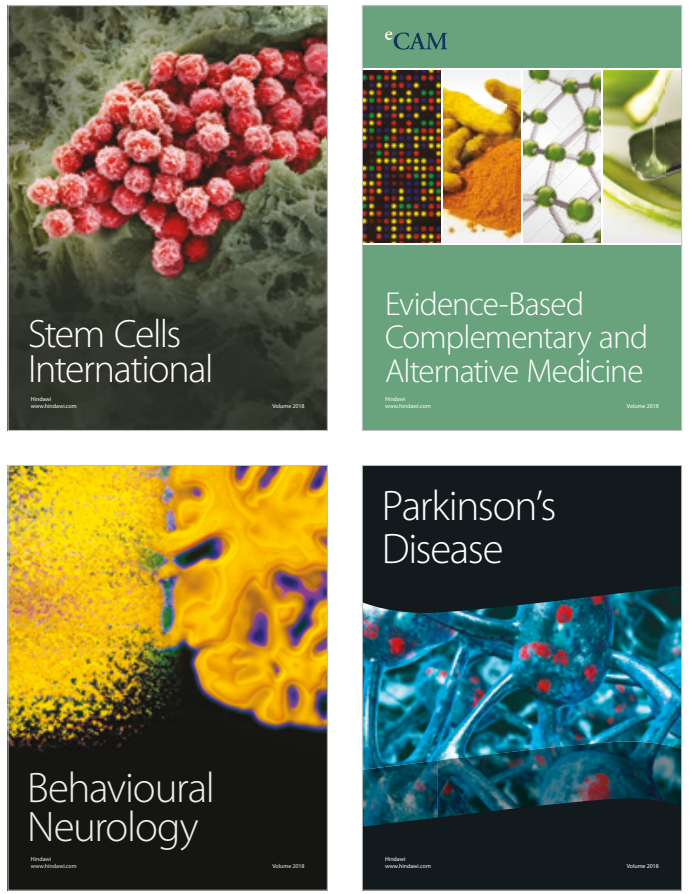

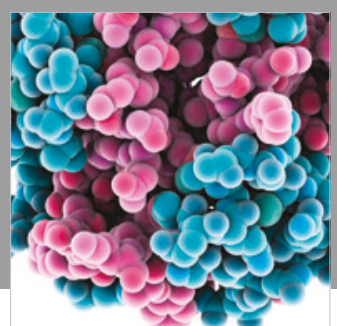

ournal of

Diabetes Research

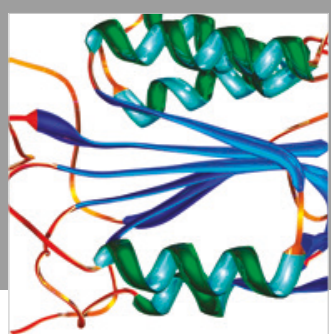

Disease Markers
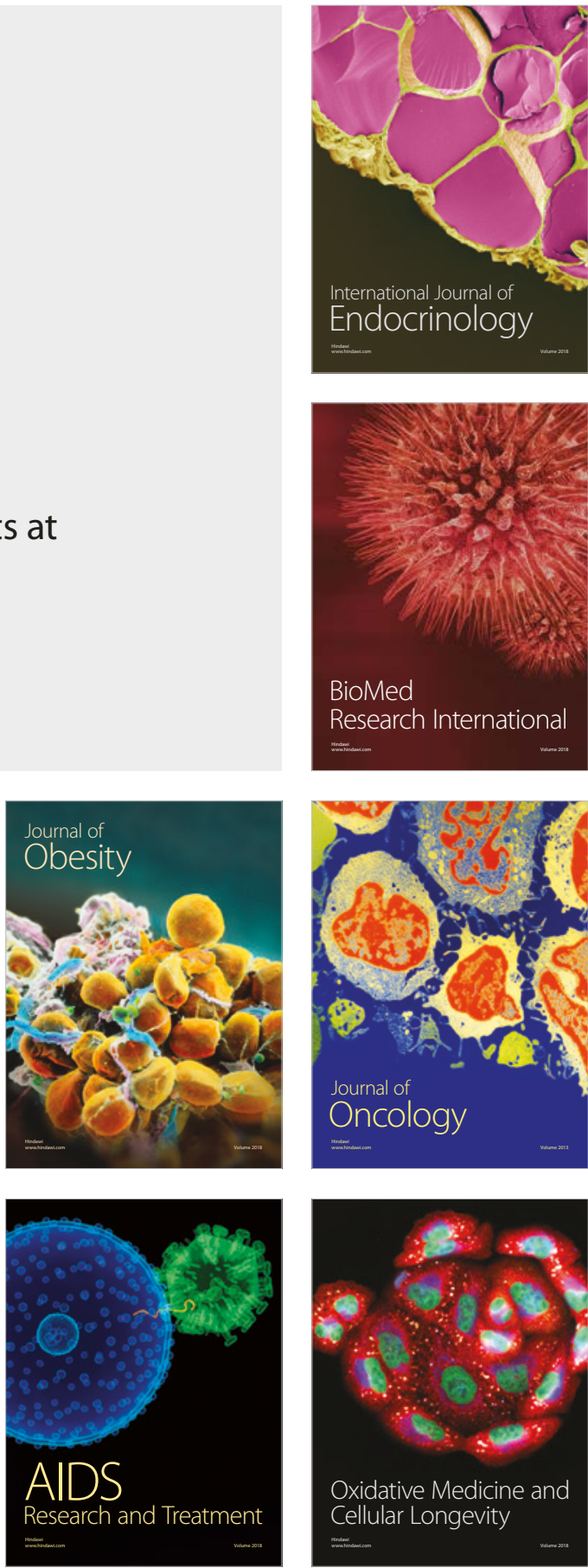